\title{
Status, results and prospects from the MoEDAL experiment at LHC
}

\author{
Vasiliki A. Mitsou* on behalf of the MoEDAL Collaboration \\ Instituto de Física Corpuscular (IFIC), CSIC - Universitat de València, \\ Parc Científic de la U.V., C/ Catedrático José Beltrán 2, \\ E-46980 Paterna (Valencia), Spain \\ E-mail: vasiliki.mitsou@ific.uv.es
}

\begin{abstract}
The MoEDAL experiment at the LHC is optimised to detect highly ionising particles such as magnetic monopoles, dyons and (multiply) electrically charged stable massive particles predicted in a number of theoretical scenarios. MoEDAL, deployed in the LHCb cavern, combines passive nuclear track detectors with magnetic monopole trapping (MMT) volumes, while spallation-product backgrounds are being monitored with an array of MediPix pixel detectors. In this paper, an introduction to the detector concept and its physics reach, complementary to that of the large general purpose LHC experiments ATLAS and CMS, is presented. Emphasis is given to the first MoEDAL results, where the null results from a search for magnetic monopoles in MMTs exposed in 2012 LHC collisions are used to set the first LHC limits on particles with magnetic charges more than 1.5 Dirac charge. The potential to search for heavy, long-lived supersymmetric electrically-charged particles is also discussed.
\end{abstract}

Corfu Summer Institute 2016 "School and Workshops on Elementary Particle Physics and Gravity" 31 August - 23 September, 2016

Corfu, Greece

${ }^{*}$ Speaker. 


\section{Introduction}

MoEDAL (Monopole and Exotics Detector at the LHC) [1, 2], the $7^{\text {th }}$ experiment at the Large Hadron Collider (LHC) [3], was approved by the CERN Research Board in 2010. It is designed to search for manifestations of new physics through highly-ionising particles in a manner complementary to ATLAS and CMS [4]. The most important motivation for the MoEDAL experiment is to pursue the quest for magnetic monopoles and dyons at LHC energies. Nonetheless the experiment is also designed to search for any massive, stable or long-lived, slow-moving particles [5] with single or multiple electric charges arising in many scenarios of physics beyond the Standard Model (SM). A selection of the physics goals and their relevance to the MoEDAL experiment are described here and elsewhere [6]. For an extended and detailed account of the MoEDAL discovery potential, the reader is referred to the MoEDAL Physics Review [7]. Emphasis is given here on recent MoEDAL results, based on the exposure of magnetic monopole trapping volumes to 8-TeV proton-proton collisions.

The structure of this paper is as follows. Section 2 provides a brief description of the MoEDAL detector. Magnetic monopoles and monopolia are briefly discussed in Section 3, whilst Section 4 presents the MoEDAL results on monopole searches. Section 5.1 is dedicated to supersymmetric models predicting massive (meta)stable states. Scenarios with doubly-charged Higgs bosons and their observability in MoEDAL are highlighted in Section 5.2. Highly-ionising exotic structures in models with extra spatial dimensions, namely microscopic black holes and D-matter, relevant to MoEDAL are briefly mentioned in Sections 5.3 and Sections 5.4, respectively. The paper concludes with a summary and an outlook in Section 6.

\section{The MoEDAL detector}

The MoEDAL detector [2] is deployed around the intersection region at Point 8 of the LHC in the LHCb experiment Vertex Locator (VELO) [8] cavern. A three-dimensional depiction of the MoEDAL experiment is presented in Fig. 1. It is a unique and largely passive LHC detector comprised of four sub-detector systems.

\subsection{Low-threshold nuclear track detectors}

The main sub-detector system is made of a large array of CR39 $($, Makrofol $(\mathrm{R}$ and Lexan $\mathbb{R}$ nuclear track detector (NTD) stacks surrounding the intersection area. The passage of a highlyionising particle through the plastic detector is marked by an invisible damage zone along the trajectory. The damage zone is revealed as a cone-shaped etch-pit when the plastic detector is etched using a hot sodium hydroxide solution. Then the sheets of plastics are scanned looking for aligned etch pits in multiple sheets. The MoEDAL NTDs have a threshold of $Z / \beta \sim 5$, where $Z$ is the charge and $\beta=v / c$ the velocity of the incident particle. In proton-proton collision running, the only source of known particles that are highly ionising enough to leave a track in MoEDAL NTDs are spallation products with range that is typically much less than the thickness of one sheet of the NTD stack. In that case the ionising signature will be that of a very low-energy electrically-charged stopped particle. This signature is distinct to that of a penetrating electrically or magnetically charged particle that will usually traverse every sheet in a MoEDAL NTD stack, 


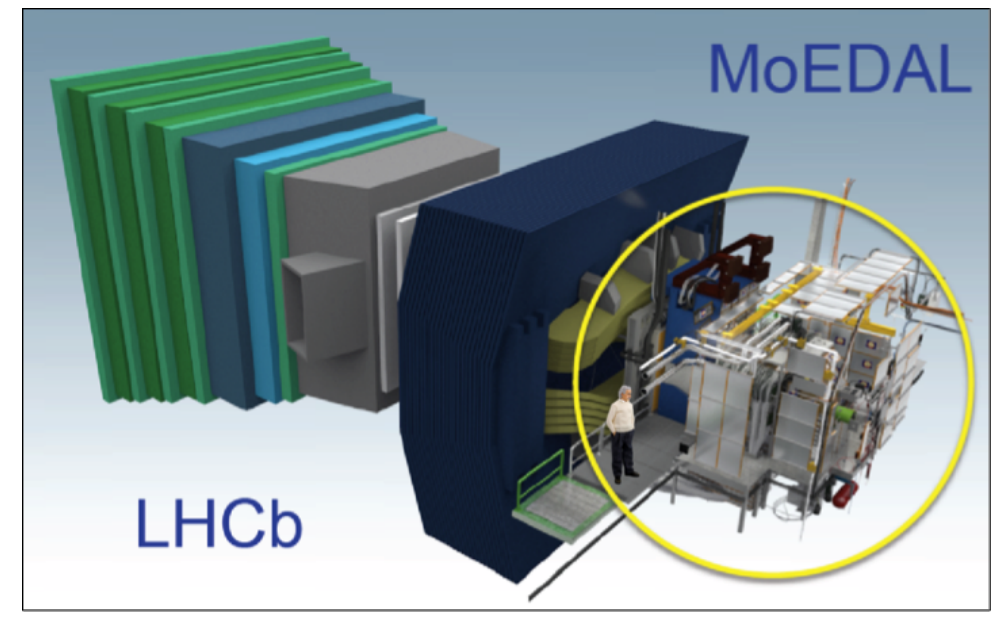

Figure 1: A three-dimensional schematic view of the MoEDAL detector (on the right) around the LHCb VELO region at Point 8 of the LHC.

accurately demarcating a track that points back to the collision point with a resolution of $\sim 1 \mathrm{~cm}$. The part of the Run-2 NTD deployment which rests on top of the LHCb VELO is visible in Fig. 2. This is the closest possible location to the interaction point and represents a novelty of this run with respect to earlier installations during Run-1.

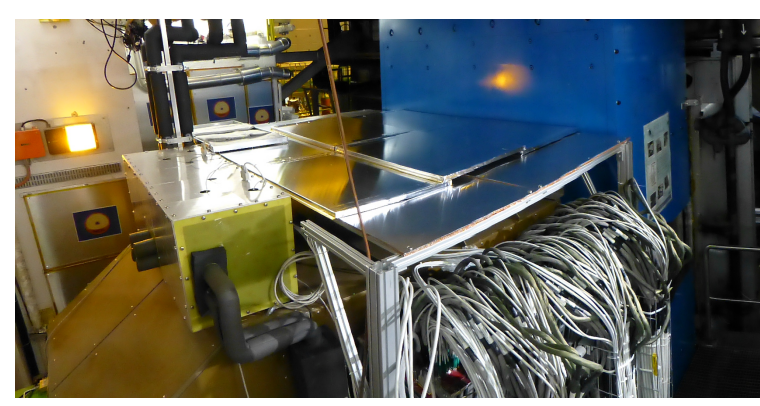

Figure 2: Part of the Run II NTD deployment on top of the LHCb VELO.

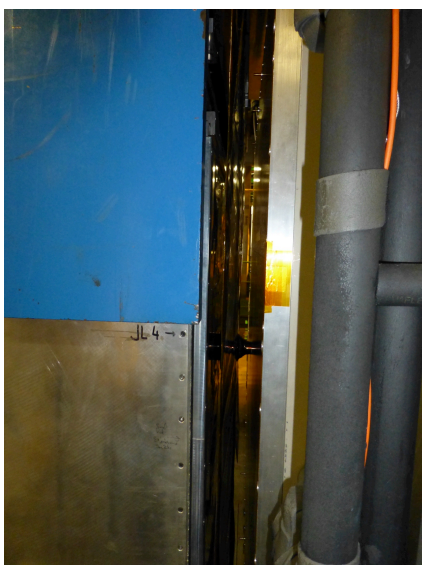

Figure 3: The VHCC between RICH1 and TT installed for Run II.

\subsection{Very high-charge catcher}

Another new feature of the Run-2 deployment is the installation of a high-threshold NTD array $(Z / \beta \sim 50)$ : the Very High Charge Catcher (VHCC). The VHCC subdetector, consisting

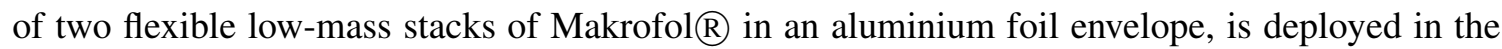
forward acceptance of the LHCb experiment between the LHCb RICH1 detector and the Trigger Tracker (TT), as shown in Fig. 3. It is the only NTD (partly) covering the forward region, adding 
only $\sim 0.5 \%$ to the $\mathrm{LHCb}$ material budget while enhancing considerably the overall geometrical coverage of MoEDAL NTDs.

\subsection{Magnetic trappers}

A unique feature of the MoEDAL detector is the use of paramagnetic magnetic monopole trappers (MMTs) to capture electrically- and magnetically-charged highly-ionising particles. Such volumes installed in IP8 for the 2015 proton-proton collisions is shown in Fig. 4. The aluminium absorbers of MMTs are subject to an analysis looking for magnetically-charged particles at a remote SQUID magnetometer facility $[9,10]$. The search for the decays of long-lived electrically charged particles that are stopped in the trapping detectors will subsequently be carried out at a remote underground facility.

A trapping detector prototype was exposed to $8 \mathrm{TeV}$ proton-proton collisions for an integrated luminosity of $0.75 \mathrm{fb}^{-1}$ in 2012. It comprised an aluminium volume consisting of 11 boxes each containing 18 cylindrical rods of $60 \mathrm{~cm}$ length and $2.5 \mathrm{~cm}$ diameter. The results of the analysing this MMTs, interpreted in terms of monopole mass and magnetic charge, are presented in Section 4.

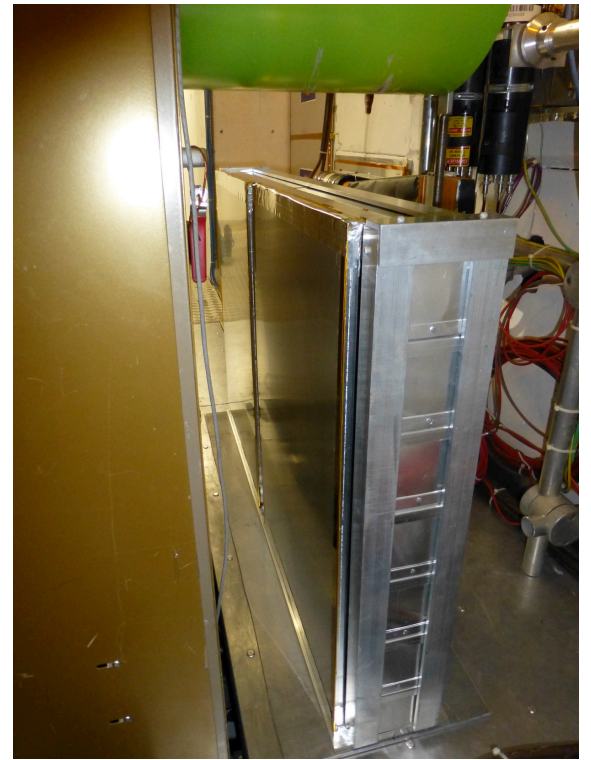

Figure 4: Deployment of the MMT for the LHC Run II.

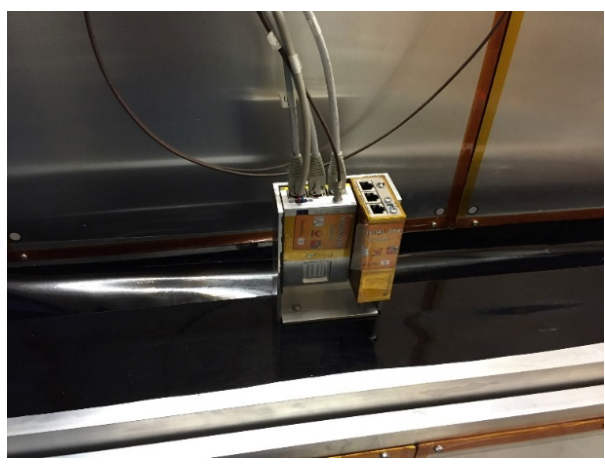

Figure 5: Run II deployment of TimePix chips in MoEDAL.

\subsection{Timepix radiation monitors}

The only non-passive MoEDAL sub-detector system comprises an array of TimePix pixel device arrays $(256 \times 256$ square pixels with a pitch of $55 \mu \mathrm{m})$ distributed throughout the MoEDAL cavern at IP8, forming a real-time radiation monitoring system of highly-ionising beam-related backgrounds. A photo of its readout setup for the 2015 installations is shown in Fig. 5. Each pixel of the innovative TimePix chip comprises a preamplifier, a discriminator with threshold adjustment, synchronisation logic and a 14-bit counter. The operation of TimePix in time-over-threshold mode allows a 3D mapping of the charge spreading effect in the whole volume of the silicon sensor, 
thus differentiating between different types of particles species from mixed radiation fields and measuring their energy deposition [11].

\section{Magnetic monopoles}

The MoEDAL detector is designed to fully exploit the energy-loss mechanisms of magnetically charged particles [12-15] in order to optimise its potential to discover these messengers of new physics. There are various theoretical scenarios in which magnetic charge would be produced at the LHC [7]: (light) 't Hooft-Polyakov monopoles [14, 16], electroweak monopoles [17 - 19], global monopoles [20-24] and monopolium [13, 25-27]. Magnetic monopoles that carry a nonzero magnetic charge and dyons possessing both magnetic and electric charge are among the most fascinating hypothetical particles. Even though there is no generally acknowledged empirical evidence for their existence, there are strong theoretical reasons to believe that they do exist, and they are predicted by many theories including grand unified theories and superstring theory [28, 29].

The theoretical motivation behind the introduction of magnetic monopoles is the symmetrisation of the Maxwell's equations and the explanation of the charge quantisation [12]. Dirac showed that the mere existence of a monopole in the universe could offer an explanation of the discrete nature of the electric charge, leading to the Dirac Quantisation Condition (DQC),

$$
\alpha g=\frac{N}{2} e, \quad N=1,2, \ldots,
$$

where $e$ is the electron charge, $\alpha=\frac{e^{2}}{4 \pi \hbar c \varepsilon_{0}}=\frac{1}{137}$ is the fine structure constant (at zero energy, as appropriate to the fact that the quantisation condition of Dirac pertains to long (infrared) distances from the centre of the monopole), $\varepsilon_{0}$ is the vacuum permittivity, and $g$ is the monopole magnetic charge. In Dirac's formulation, magnetic monopoles are assumed to exist as point-like particles and quantum mechanical consistency conditions lead to Eq. (3.1), establishing the value of their magnetic charge. Although monopoles symmetrise Maxwell's equations in form, there is a numerical asymmetry arising from the DQC, namely that the basic magnetic charge is much larger than the smallest electric charge. A magnetic monopole with a single Dirac charge $\left(g_{\mathrm{D}}\right)$ has an equivalent electric charge of $\beta(137 e / 2)$. Thus for a relativistic monopole the energy loss is around 4,700 times $\left(68.5^{2}\right)$ that of a minimum-ionising electrically-charged particle. The monopole mass remains a free parameter of the theory.

A possible explanation for the lack of experimental confirmation of monopoles is Dirac's proposal $[12,13,25]$ that monopoles are not seen freely because they form a bound state called monopolium [26, 27, 30] being confined by strong magnetic forces. Monopolium is a neutral state, hence it is difficult to detect directly at a collider detector, although its decay into two photons would give a rather clear signal for the ATLAS and CMS detectors [31, 32], which however would not be visible in the MoEDAL detector. Nevertheless according to a novel proposal [33], the LHC radiation detector systems can be used to turn the LHC itself into a new physics search machine by detecting final-state protons $p p \rightarrow p X p$ exiting the LHC beam vacuum chamber at locations determined by their fractional momentum losses. Such technique would be appealing for detecting monopolia. Furthermore the monopolium might break up in the medium of MoEDAL into highlyionising dyons, which subsequently can be detected in MoEDAL [7]. Moreover its decay via 
photon emission would produce a peculiar trajectory in the medium, should the decaying states are also magnetic multipoles [7].

\section{Searches for monopoles in MoEDAL}

The high ionisation of slow-moving magnetic monopoles and dyons, implies quite characteristic trajectories when such particles interact with the MoEDAL NTDs, which can be revealed during the etching process $[2,7]$. In addition, the high magnetic charge of a monopole (which is expected to be at least one Dirac charge $g_{D}=68.5 e$ ( $c f$. Eq. (3.1)) implies a strong magnetic dipole moment, which in turn may result in a strong binding of the monopole with the ${ }_{13}^{27} \mathrm{Al}$ nuclei of the aluminium MoEDAL MMTs. In such a case, the presence of a monopole trapped in an aluminium bar of an MMT would de detected through the existence of a persistent current, defined as the difference between the currents in the SQUID of a magnetometer before and after the passage of the bar through the sensing coil.

In the context of MoEDAL searches, a prototype MoEDAL trapping detector samples was exposed to $8-\mathrm{TeV}$ proton-proton collisions at the $\mathrm{LHC}$, for an integrated luminosity of $0.75 \mathrm{fb}^{-1}$ during Run I. No magnetic charge exceeding $0.5 g_{\mathrm{D}}$ was detected in any of the exposed samples, allowing limits to be placed on monopole production in the mass range $100 \mathrm{GeV} \leq m \leq 3500 \mathrm{GeV}$ [34]. Model-independent cross-section limits have been presented in fiducial regions of monopole energy and direction for $1 g_{\mathrm{D}} \leq|g| \leq 6 g_{\mathrm{D}}$, and model-dependent cross-section limits are obtained for Drell-Yan (DY) pair production of spin-1/2 and spin-0 monopoles for $1 g_{\mathrm{D}} \leq|g| \leq 4 g_{\mathrm{D}}(c f$. Fig. 6). Under the assumption of Drell-Yan cross sections, mass limits are derived for $|g|=2 g_{\mathrm{D}}$ and $|g|=3 g_{\mathrm{D}}$ for the first time at the LHC, surpassing previous results from ATLAS Collaboration [35, 36], which placed limits only for monopoles with magnetic charge $|g|=1 g_{\mathrm{D}}$. Caution, however, should be exerted here in the sense that the non-perturbative nature of the large magnetic Dirac charge of the monopole invalidate any perturbative treatment based on Drell-Yan calculations of the pertinent cross sections and hence any result based on the latter is only indicative, due to the lack of any other concrete theoretical treatment. New results with MMTs exposed at 13$\mathrm{TeV} p p$ LHC collisions have been published recently [37] extending further the limits in terms of monopole mass and charge up to $5 g_{\mathrm{D}}$ in DY processes. A comparison of the limits on monopole production cross sections set by other colliders with those set by MoEDAL is given in Fig. 7 [29], while general limits including searches in cosmic radiation are reviewed in Ref. [38].

\section{Beyond magnetic monopoles}

\subsection{Electrically-charged long-lived particles in supersymmetry}

Supersymmetry (SUSY) is an extension of the Standard Model which assigns to each SM field a superpartner field with a spin differing by a half unit. SUSY provides elegant solutions to several open issues in the SM, such as the hierarchy problem, the identity of dark matter, and the grand unification. SUSY scenarios propose a number of massive slowly moving $(\beta \lesssim 0.5)$ electrically charged particles. If they are sufficiently long-lived to travel a distance of at least $\mathscr{O}(1 \mathrm{~m})$ before decaying and their $Z / \beta \gtrsim 0.5$, then they will be detected in the MoEDAL NTDs. No highly-charged particles are expected in such a theory, but there are several scenarios in which supersymmetry 

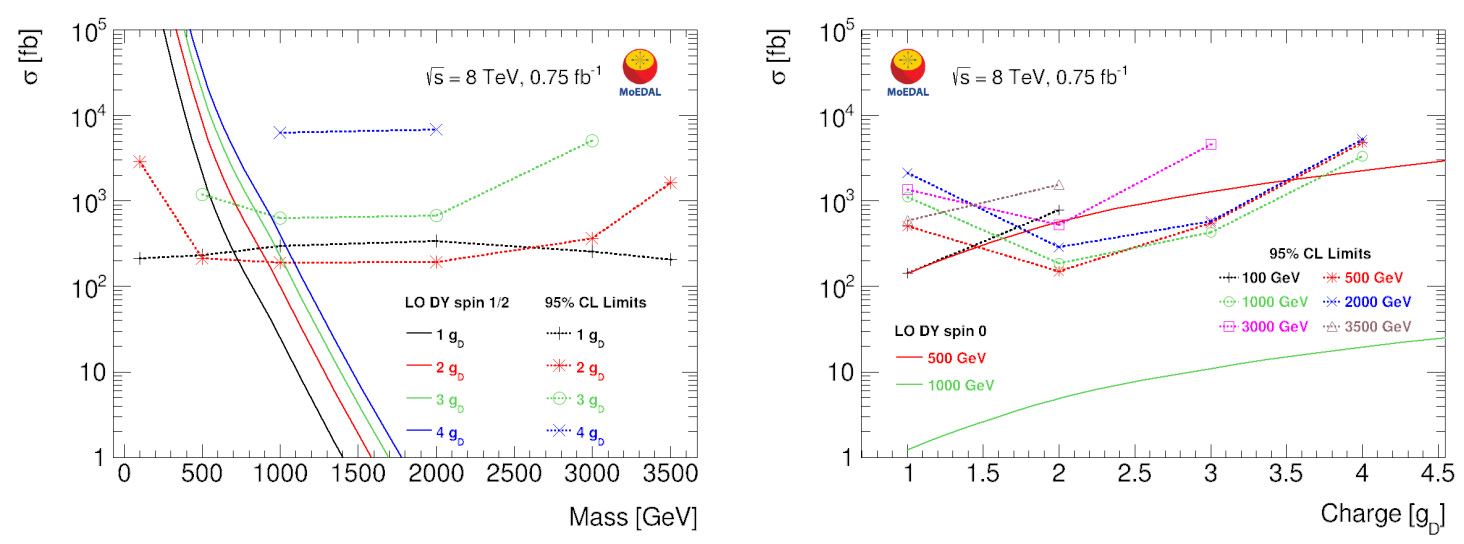

Figure 6: Cross-section upper limits at $95 \%$ confidence level for DY monopole production as a function of mass for spin-1/2 (left) and as a function of magnetic charge for spin-0 monopoles (right). The various line styles correspond to different monopole charges (left) or masses (right). The solid lines are DY cross-section calculations at leading order. From Ref [34].

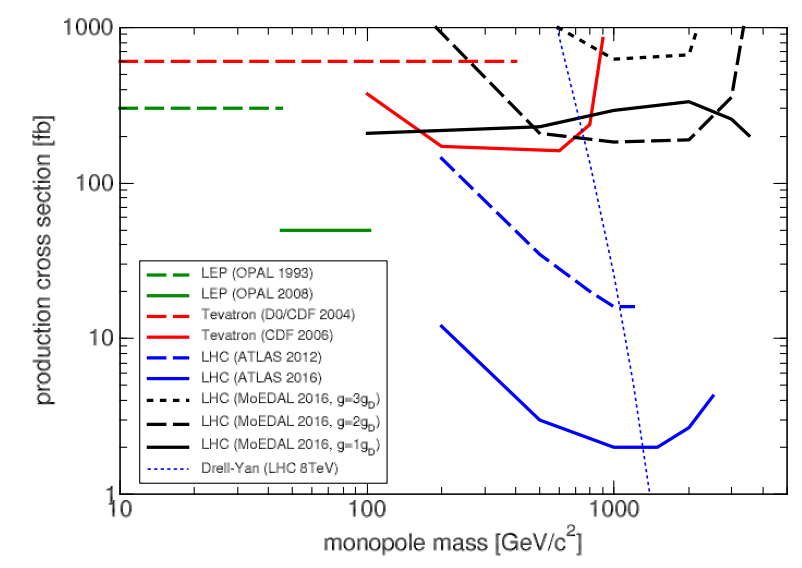

Figure 7: Upper bounds on the monopole production cross sections in various colliders, including MoEDAL. From Ref. [29].

may yield massive, long-lived particles that could have electric charges \pm 1 , potentially detectable in MoEDAL if they are produced with low velocities.

The lightest supersymmetric particle (LSP) is stable in models where $R$ parity is conserved. The LSP should have no strong or electromagnetic interactions, for otherwise it would bind to conventional matter and be detectable in anomalous heavy nuclei [39]. Possible weakly-interacting neutral candidates in the Minimal Supersymmetric Standard Model (MSSM) include the sneutrino, which has been excluded by LEP and direct searches, the lightest neutralino $\tilde{\chi}_{1}^{0}$ (a mixture of spartners of the $Z, H$ and $\gamma$ ) and the gravitino $\tilde{G}$. 


\subsubsection{Supersymmetric scenarios with $R$-parity violation}

Several scenarios featuring metastable charged sparticles might be detectable in MoEDAL. One such scenario is that $R$ parity may not be exact, since there is no exact local symmetry associated with either $L$ or $B$, and hence no fundamental reason why they should be conserved. One could consider various ways in which $L$ and/or $B$ could be violated in such a way that $R$ is violated, as represented by the following superpotential terms:

$$
W_{R V}=\lambda_{i j k}^{\prime \prime} \bar{U}_{i} \bar{D}_{j} \bar{D}_{k}+\lambda_{i j k}^{\prime} L_{i} Q_{j} \bar{D}_{k}+\lambda_{i j k} L_{i} L_{j} \bar{E}_{k}+\mu_{i} L_{i} H,
$$

where $Q_{i}, \bar{U}_{i}, \bar{D}_{i}, L_{i}$ and $\bar{E}_{i}$ denote chiral superfields corresponding to quark doublets, antiquarks, lepton doublets and antileptons, respectively, with $i, j, k$ generation indices. The simultaneous presence of terms of the first and third type in Eq. (5.1), namely $\lambda$ and $\lambda^{\prime \prime}$, is severely restricted by lower limits on the proton lifetime, but other combinations are less restricted. The trilinear couplings in Eq. (5.1) generate sparticle decays such as $\tilde{q} \rightarrow \bar{q} \bar{q}$ or $q \ell$, or $\tilde{\ell} \rightarrow \ell \ell$, whereas the bilinear couplings in Eq. (5.1) generate Higgs-slepton mixing and thereby also $\tilde{q} \rightarrow q \ell$ and $\tilde{\ell} \rightarrow \ell \ell$ decays [40]. For a nominal sparticle mass $\sim 1 \mathrm{TeV}$, the lifetime for such decays would exceed a few nanoseconds for $\lambda, \lambda^{\prime}, \lambda^{\prime \prime}<10^{-8}$.

If $R$ parity is broken, the LSP would be unstable, and might be charged and/or coloured. In the former case, it might be detectable directly at the LHC as a massive slowly-moving charged particle. In the latter case, the LSP would bind with light quarks and/or gluons to make colour-singlet states, the so-called $R$-hadrons, and any charged state could again be detectable as a massive slowlymoving charged particle. If $\lambda \neq 0$, the prospective experimental signature would be similar to a stau next-to-lightest sparticle (NLSP) case to be discussed later. On the other hand, if $\lambda^{\prime}$ or $\lambda^{\prime \prime} \neq 0$, the prospective experimental signature would be similar to a stop NLSP case, yielding the possibility of charge-changing interactions while passing through matter. This could yield a metastable charged particle, created whilst passing through the material surrounding the intersection point, that would be detected by MoEDAL.

\subsubsection{Metastable lepton NLSP in the CMSSM with a neutralino LSP}

However, even if $R$ parity is exact, the NLSP may be long lived. This would occur, for example, if the LSP is the gravitino, or if the mass difference between the NLSP and the neutralino LSP is small, offering more scenarios for long-lived charged sparticles. In neutralino dark matter scenarios based on the constrained MSSM (CMSSM), for instance, the most natural candidate for the NLSP is the lighter stau slepton $\tilde{\tau}_{1}$ [41], which could be long lived if $m_{\tilde{\tau}_{1}}-m_{\tilde{\chi}_{1}^{0}}$ is small. There are several regions of the CMSSM parameter space that are compatible with the constraints imposed by unsuccessful searches for sparticles at the LHC, as well as the discovered Higgs boson mass. These include a strip in the focus-point region where the relic density of the LSP is brought down into the range allowed by cosmology because of its relatively large Higgsino component, a region where the relic density is controlled by rapid annihilation through direct-channel heavy Higgs resonances, and a strip where the relic LSP density is reduced by coannihilations with neardegenerate staus and other sleptons. It was found in a global analysis that the two latter possibilities are favoured [42].

In the coannihilation region of the CMSSM, the lighter $\tilde{\tau}_{1}$ is expected to be the lightest slepton [41], and the $\tilde{\tau}_{1}-\tilde{\chi}_{1}^{0}$ mass difference may well be smaller than $m_{\tau}$ : indeed, this is required at 
large LSP masses. In this case, the dominant stau decays for $m_{\tilde{\tau}_{1}}-m_{\tilde{\chi}_{1}^{0}}>160 \mathrm{MeV}$ are expected to be into three particles: $\tilde{\chi}_{1}^{0} v \pi$ or $\tilde{\chi}_{1}^{0} v \rho$. If $m_{\tilde{\tau}_{1}}-m_{\tilde{\chi}_{1}^{0}}<1.2 \mathrm{GeV}$, the $\tilde{\tau}_{1}$ lifetime is calculated to be so long, in excess of $\sim 100 \mathrm{~ns}$, that it is likely to escape the detector before decaying, and hence would be detectable as a massive, slowly-moving charged particle [43, 44]. The relevance of such scenarios while considering cosmological constraints is demonstrated in Fig. 8. Even is lepton-flavour violating couplings are allowed, the long lifetime of the staus remains [44].

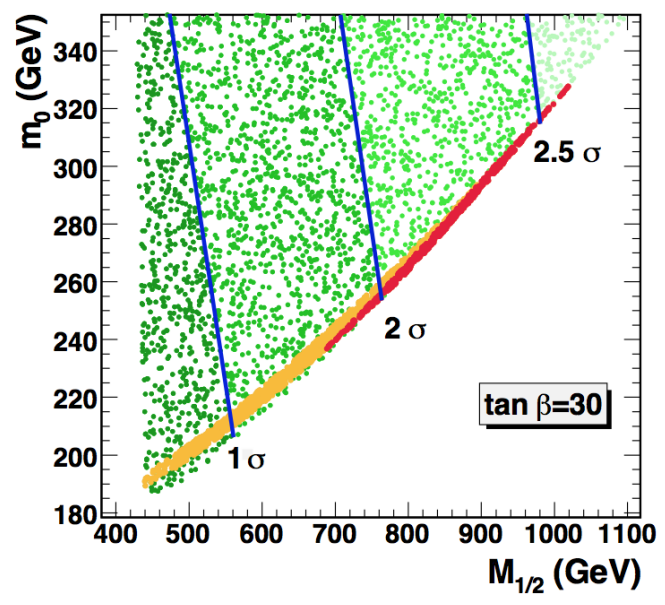

Figure 8: Allowed parameter regions in $M_{1 / 2}-m_{0}$ plane fixing $A_{0}=600 \mathrm{GeV}$ and $\tan \beta=30$. The red (dark) narrow band is consistent region with dark matter abundance and $\delta m<m_{\tau}$ and the yellow (light) narrow band is that with $\delta m>m_{\tau}$. The green regions are inconsistent with the dark matter abundance, and in the white (excluded) area the LSP is the stau. The favoured regions of the muon anomalous magnetic moment at $1 \sigma, 2 \sigma$ and $2.5 \sigma$ confidence level are indicated by solid lines. From Ref. [44].

\subsubsection{Metastable sleptons in gravitino LSP scenarios}

On the other hand, in gravitino dark matter scenarios with more general options for the pattern of supersymmetry breaking, other options appear quite naturally, including the lighter selectron or smuon, or a sneutrino [45], or the lighter stop squark $\tilde{t}_{1}$ [46]. If the gravitino $\tilde{G}$ is the LSP, the decay rate of a slepton NLSP is given by

$$
\Gamma(\tilde{\ell} \rightarrow \tilde{G} \ell)=\frac{1}{48 \pi M_{*}^{2}} \frac{m_{\tilde{\ell}}^{5}}{M_{\tilde{G}}^{2}}\left[1-\frac{M_{\tilde{G}}^{2}}{m_{\tilde{\ell}}^{2}}\right]^{4},
$$

where $M_{*}$ is the Planck scale. Since $M_{*}$ is much larger than the electroweak scale, the NLSP lifetime is naturally very long.

Gravitino (or axino) LSP with a long-lived charged stau may arise in gauge mediation and minimal supergravity models [47]. Large part of the parameter space potentially attractive for long-lived slepton searches with MoEDAL are compatible with cosmological constraints on the dark-matter abundance in superweakly interacting massive particle scenarios [48].

\subsubsection{Long-lived gluinos in split supersymmetry}

The above discussion has been in the context of the CMSSM and similar scenarios where all the supersymmetric partners of Standard Model particles have masses in the TeV range. Another scenario is "split supersymmetry", in which the supersymmetric partners of quarks and leptons are very heavy, of a scale $m_{s}$, whilst the supersymmetric partners of SM bosons are relatively light [49]. In such a case, the gluino could have a mass in the $\mathrm{TeV}$ range and hence be accessible to the $\mathrm{LHC}$, 
but would have a very long lifetime:

$$
\tau \approx 8\left(\frac{m_{s}}{10^{9} \mathrm{GeV}}\right)^{4}\left(\frac{1 \mathrm{TeV}}{m_{\tilde{g}}}\right)^{5} \mathrm{~s} .
$$

Long-lived gluinos would form long-lived gluino R-hadrons including gluino-gluon (gluinoball) combinations, gluino- $q \bar{q}$ (mesino) combinations and gluino- $q q q$ (baryino) combinations. The heavier gluino hadrons would be expected to decay into the lightest species, which would be metastable, with a lifetime given by Eq. (5.3), and it is possible that this metastable gluino hadron could be charged.

In the same way as stop hadrons, gluino hadrons may flip charge through conventional strong interactions as they pass through matter, and it is possible that one may pass through most of a conventional LHC tracking detector undetected in a neutral state before converting into a metastable charged state that could be detected by MoEDAL.

\subsubsection{Experimental considerations}

There are several considerations supporting the complementary aspects of MoEDAL w.r.t. ATLAS and CMS when discussing the observability of (meta-)stable massive electrically-charged particles. Most of them stem from MoEDAL being "time-agnostic" due to the passive nature of its detectors. Therefore signal from very slowly moving particles will not be lost due to arriving in several consecutive bunch crossings. ATLAS and CMS, on the other hand, perform triggeredbased analyses relying either on triggering on accompanying "objects", e.g. missing transverse energy, or by developing and deploying specialised triggers. In both cases the efficiency may lower and in the former the probed parameter space may be reduced. MoEDAL is mainly limited by the geometrical acceptance of the detectors, especially the MMTs, and by the requirement of passing the $Z / \beta$ threshold of NTDs. In general ATLAS and CMS have demonstrated to cover high-velocities $\beta \gtrsim 0.2$, while MoEDAL is sensitive to lower ones $\beta \lesssim 0.2$.

When discussing the detection of particles stopped (trapped) in material that they may decay later, different possibilities are explored. CMS and ATLAS look in empty bunch crossings for decays of trapped particles into jets. MoEDAL MMTs may be monitored in a underground/basement laboratory for tracks arising from such decays. The background in the latter case, coming from cosmic rays, should be easier to control and assess. The probed lifetimes should be larger due to the unlimited monitoring time.

\subsection{Doubly-charged Higgs bosons}

Doubly-charged particles appear in many theoretical scenarios beyond the SM. For example, doubly-charged scalar states, usually termed doubly-charged Higgs fields, appear in left-right symmetric models [50 - 52] and in see-saw models for neutrino masses with Higgs triplets. A number of models encompasses additional symmetries and extend the SM Higgs sector by introducing doubly-charged Higgs bosons. A representative example of such a model is the L-R Symmetric Model (LRSM) [50-52], proposed to amend the fact that the weak-interaction couplings are strictly left handed by extending the gauge group of the SM so as to include a right-handed sector. The simplest realisation is an LRSM $[50,51]$ that postulates a right-handed version of the weak interaction, whose gauge symmetry is spontaneously broken at high mass scale, leading to 
the parity-violating SM. This model naturally accommodates recent data on neutrino oscillations and the existence of small neutrino masses. The model generally requires Higgs triplets containing doubly-charged Higgs bosons $\left(H^{ \pm \pm}\right) \Delta_{R}^{++}$and $\Delta_{L}^{++}$, which could be light in the minimal supersymmetric left-right model [53].

Single production of a doubly-charged Higgs boson at the LHC proceeds via vector boson fusion, or through the fusion of a singly-charged Higgs boson with either a $W^{ \pm}$or another singlycharged Higgs boson. The amplitudes of the $W_{L} W_{L}$ and $W_{R} W_{R}$ vector boson fusion processes are proportional to $v_{L, R}$, the vacuum expectation values of the neutral members of the scalar triplets of the LRSM. For the case of $\Delta_{R}^{++}$production, the vector boson fusion process dominates. Pair production of doubly-charged Higgs bosons is also possible via a Drell-Yan process, with $\gamma, Z$ or $Z_{R}$ exchanged in the $s$-channel, but at a high kinematic price since substantial energy is required to produce two heavy particles. In the case of $\Delta_{L}^{++}$, double production may nevertheless be the only possibility if $v_{L}$ is very small or vanishing.

The decay of a doubly-charged Higgs boson can proceed via several channels. The dilepton signature leads to the (experimentally clean) final state $q \bar{q} \rightarrow \Delta_{L}^{++} \Delta_{L}^{--} \rightarrow 4 \ell$. However as long as the triplet vacuum expectation value, $v_{\Delta}$, is much larger than $10^{-4} \mathrm{GeV}$, the doubly-charged Higgs decay predominantly into a pair of same-sign $W$ bosons. For very small Yukawa couplings $H_{\ell \ell} \lesssim 10^{-8}$, the doubly-charged Higgs boson can be quasi-stable [54]. In Fig. 9, the partial decay width of the doubly charged Higgs boson into a $W$ boson pair is shown as a function of its mass. For $v_{\Delta} \gg 10^{-4} \mathrm{GeV}$, this partial width is roughly equal to the total width of the doubly charged Higgs boson. In the case of long lifetimes, slowly moving pseudo-stable Higgs bosons could be detected in the MoEDAL NTDs. For example with CR39, one could detect doubly-charged Higgs particles moving with speeds less than around $\beta \simeq 0.4$.

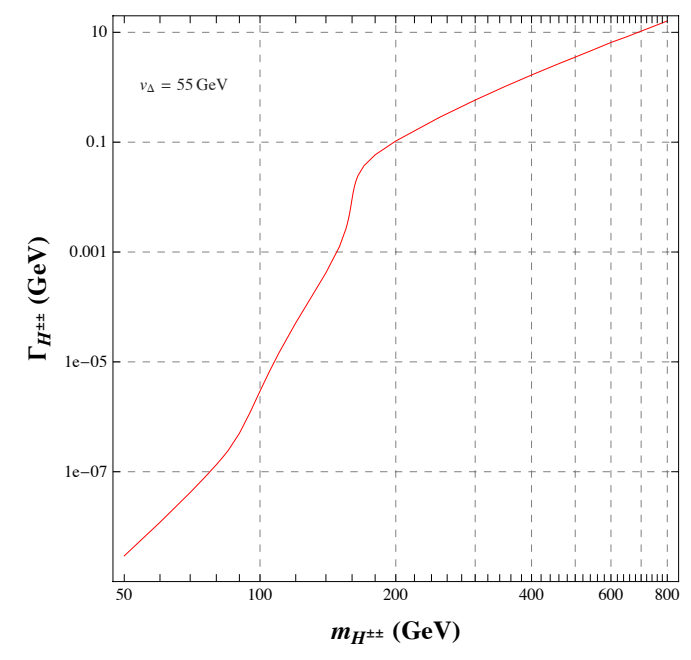

Figure 9: Partial decay width of $H^{ \pm \pm} \rightarrow$ $W^{ \pm} W^{ \pm}$as a function of $m_{H^{ \pm \pm}}$for $v_{\Delta}=$ $55 \mathrm{GeV}$. From Ref. [54].

\subsection{Black hole remnants in large extra dimensions}

Over the last decades, models based on compactified extra spatial dimensions (ED) have been proposed in order to explain the large gap between the electroweak (EW) and the Planck scale of $M_{\mathrm{EW}} / M_{\mathrm{PL}} \approx 10^{-17}$. The four main scenarios relevant for searches at LHC the Arkani-HamedDimopoulos-Dvali (ADD) model of large extra dimensions [55], the Randall-Sundrum (RS) model 
of warped extra dimensions [56], $\mathrm{TeV}^{-1}$-sized extra dimensions [57], and the Universal Extra Dimensions (UED) model [58].

The existence of extra spatial dimensions $[55,56]$ and a sufficiently small fundamental scale of gravity open up the possibility that microscopic black holes be produced and detected [59-64] at the LHC. Once produced, the black holes will undergo an evaporation process categorised in three stages [59, 60]: the balding phase, the actual evaporation phase, and finally the Planck phase. It is generally assumed that the black hole will decay completely to some last few SM particles. However, another intriguing possibility is that the remaining energy is carried away by a stable remnant.

The prospect of microscopic black hole production at the LHC within the framework of models with large extra dimensions has been studied in Ref. [55]. Black holes produced at the LHC are expected to decay with an average multiplicity of $\sim 10-25$ into SM particles, most of which will be charged, though the details of the multiplicity distribution depend on the number of extra dimensions [65]. After the black holes have evaporated off enough energy to reach the remnant mass, some will have accumulated a net electric charge. According to purely statistical considerations, the probability for being left with highly-charged black hole remnants drops fast with the deviation from the average. The largest fraction of the black holes should have charges \pm 1 or zero, although a smaller but non-negligible fraction would be multiply charged.

The fraction of charged black-hole remnants has been estimated $[65,66]$ using the PYTHIA event generator [67] and the CHARYBDIS program [64]. It was assumed that the effective temperature of the black hole drops towards zero for a finite remnant mass, $M_{R}$. The value of $M_{R}$ does not noticeably affect the investigated charge distribution, as it results from the very general statistical distribution of the charge of the emitted particles.

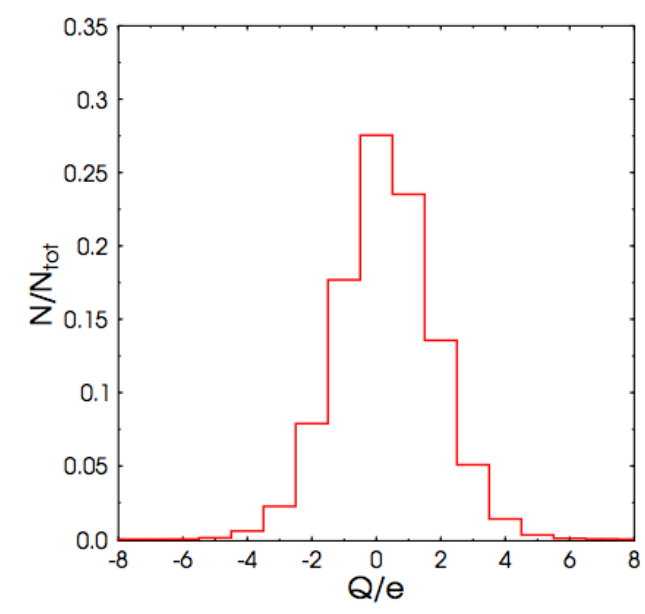

Figure 10: The distribution of black-hole remnant charges in proton-proton interactions at $\sqrt{s}=14 \mathrm{TeV}$ calculated with the PYTHIA event generator [67] and the CHARYBDIS program [64]. From Ref. [66].

Thus, independent of the underlying quantum-gravitational assumption leading to the remnant formation, it was found that about $30 \%$ of the remnants are neutral, whereas $\sim 40 \%$ would be singly-charged black holes, and the remaining $~ 30 \%$ of remnants would be multiply-charged. The distribution of the remnant charges obtained is shown in Fig. 10. The black hole remnants considered here are heavy, with masses of a TeV or more. A significant fraction of the black-hole remnants produced would have a $Z / \beta$ of greater than five, high enough to register in the CR39 
NTDs forming the LT-NTD sub-detector of MoEDAL.

\subsection{D-matter}

Some versions of string theory include higher-dimensional "domain-wall"-like membrane (brane) structures in space-time, called D-branes. In some cases the bulk is "punctured" by lowerdimensional D-brane defects, which are either point-like or have their longitudinal dimensions compactified [68]. From a low-energy observer's perspective, such structures would effectively appear to be point-like $D$-particles. The latter have dynamical degrees of freedom, thus they can be treated as quantum excitations above the vacuum $[68,69]$ collectively referred to as $D$-matter. D-matter states are non-perturbative stringy objects with masses of order $m_{D} \sim M_{s} / g_{s}$, where $g_{s}$ is the string coupling, typically of order one so that the observed gauge and gravitational couplings is reproduced. Hence, the D-matter states could be light enough to be phenomenologically relevant at the LHC.

Depending on their type, D-branes could carry integral or torsion (discrete) charges with the lightest D-particle (LDP) being stable. Therefore the LDPs are possible candidates for cold dark matter [69]. D-particles are solitonic non-perturbative objects in the string/brane theory. As discussed in the relevant literature [69], there are similarities and differences between D-particles and magnetic monopoles with non-trivial cosmological implications [68, 70-72]. An important difference is that they could have perturbative couplings, with no magnetic charge in general. Nonetheless, in the context of brane-inspired gauge theories, brane states with magnetic charges can be constructed, which would manifest themselves in MoEDAL in a manner similar to magnetic monopoles.

Non-magnetically-charged D-matter, on the other hand, could be produced at colliders and also produce interesting signals of direct relevance to the MoEDAL experiment. For instance, excited states of D-matter $\left(\mathrm{D}^{\star}\right)$ can be electrically-charged. For typical string couplings of phenomenological relevance, the first few massive levels may be accessible to the LHC. Depending on the details of the microscopic model considered, and the way the SM is embedded, such massive charged states can be relatively long-lived, and could likewise be detectable with MoEDAL. D-matter/antimatter pairs can be produced [71, 72] by the decay of intermediate off-shell Z-bosons.

\section{Summary and outlook}

MoEDAL is going to extend considerably the LHC reach in the search for (meta)stable highly ionising particles. The latter are predicted in a variety of theoretical models and include: magnetic monopoles, SUSY stable (or, rather, long-lived) spartners, quirks, strangelets, Q-balls, fractionallycharged massive particles, etc [7]. Such particles can be light enough to be producible at the LHC energies (see e.g. Q-balls in the context of some SUSY or brane models [73]). In the talk we have described searches for only a subset of those particles, due to lack of space. Specifically, we discussed monopoles, partners in some SUSY models, doubly charged Higgs bosons, black hole remnants in models with large extra spatial dimensions, as well as some (more exotic) scenarios on D-matter which characterises some brane models.

The MoEDAL design is optimised to probe precisely all such long lived states, unlike the other LHC experiments [4]. Furthermore it combines different detector technologies: plastic nu- 
clear track detectors (NTDs), trapping volumes and pixel sensors [2]. The first physics results, pertaining to magnetic monopole trapping detectors, obtained with LHC Run I data, have been published [34] and the corresponding analysis at $13 \mathrm{TeV}$ has been released recently [37]. The MoEDAL Collaboration is preparing new analyses with more Run II data, with other detectors (NTDs) and with a large variety of interpretations involving not only magnetic but also electric charges.

\section{Acknowledgments}

The author is grateful to the CORFU2016 School organisers for the kind invitation to present this talk. She acknowledges support by the Spanish Ministry of Economy and Competitiveness (MINECO) under the project FPA2015-65652-C4-1-R, by the Generalitat Valenciana through the project PROMETEO II/2013-017, by the Spanish National Research Council (CSIC) under the CT Incorporation Program 2016501002 and by the Severo Ochoa Excellence Centre Project SEV 2014-0398.

\section{References}

[1] For general information on the MoEDAL experiment, see: http://moedal.web.cern.ch/

[2] J. Pinfold et al. [MoEDAL Collaboration], "Technical Design Report of the MoEDAL Experiment," CERN-LHCC-2009-006, MoEDAL-TDR-001, and references therein.

[3] L. Evans and P. Bryant, JINST 3, S08001 (2008).

[4] A. De Roeck, A. Katre, P. Mermod, D. Milstead and T. Sloan, Eur. Phys. J. C 72, 1985 (2012).

[5] M. Fairbairn, A. C. Kraan, D. A. Milstead, T. Sjostrand, P. Z. Skands and T. Sloan, Phys. Rept. 438, 1 (2007); S. Burdin, M. Fairbairn, P. Mermod, D. Milstead, J. Pinfold, T. Sloan and W. Taylor, Phys. Rept. 582, 1 (2015).

[6] V. A. Mitsou [MoEDAL Collaboration], EPJ Web Conf. 95, 04042 (2015); PoS EPS-HEP2015, 109 (2015); J. Phys. Conf. Ser., to appear [arXiv:1703.07141 [hep-ex]]; N. E. Mavromatos and V. A. Mitsou [MoEDAL Collaboration], "Physics reach of MoEDAL at LHC: magnetic monopoles, supersymmetry and beyond," EPJ Web Conf., to appear [arXiv:1612.07012 [hep-ph]].

[7] B. Acharya et al. [MoEDAL Collaboration], Int. J. Mod. Phys. A 29, 1430050 (2014).

[8] A. A. Alves, Jr. et al. [LHCb Collaboration], JINST 3, S08005 (2008).

[9] M. D. Joergensen, A. De Roeck, H.-P. Hachler, A. Hirt, A. Katre, P. Mermod, D. Milstead and T. Sloan, "Searching for magnetic monopoles trapped in accelerator material at the Large Hadron Collider," arXiv:1206.6793 [physics.ins-det] (2012).

[10] A. De Roeck, H. P. Hächler, A. M. Hirt, M.-D. Joergensen, A. Katre, P. Mermod, D. Milstead and T. Sloan, Eur. Phys. J. C 72, 2212 (2012).

[11] A. Sopczak et al., IEEE Trans. Nucl. Sci. 62, 3225 (2015).

[12] P. A. M. Dirac, Proc. Roy. Soc. Lond. A 133, 60 (1931).

[13] P. A. M. Dirac, Phys. Rev. 74, 817 (1948). 
[14] G. 't Hooft, Nucl. Phys. B 79, 276 (1974); A. M. Polyakov, JETP Lett. 20, 194 (1974) [Pisma Zh. Eksp. Teor. Fiz. 20, 430 (1974)].

[15] B. Julia and A. Zee, Phys. Rev. D 11, 2227 (1975); Y. Nambu, Nucl. Phys. B 130, 505 (1977); E. Witten, Phys. Lett. 86B, 283 (1979); G. Lazarides, M. Magg and Q. Shafi, Phys. Lett. 97B, 87 (1980); R. d. Sorkin, Phys. Rev. Lett. 51, 87 (1983); D. J. Gross and M. J. Perry, Nucl. Phys. B 226, 29 (1983); J. S. Schwinger, Science 165, 757 (1969); J. Preskill, Ann. Rev. Nucl. Part. Sci. 34, 461 (1984); A. Achucarro and T. Vachaspati, Phys. Rept. 327, 347 (2000) [Phys. Rept. 327, 427 (2000)]; T. W. Kephart, C. A. Lee and Q. Shafi, JHEP 0701, 088 (2007); D. G. Pak, P. M. Zhang and L. P. Zou, Int. J. Mod. Phys. A 30, no. 27, 1550164 (2015); A. Rajantie, JHEP 0601, 088 (2006).

[16] V. Vento and V. S. Mantovani, "On the magnetic monopole mass,” arXiv:1306.4213 [hep-ph] (2013).

[17] Y. M. Cho and D. Maison, Phys. Lett. B 391, 360 (1997); W. S. Bae and Y. M. Cho, J. Korean Phys. Soc. 46, 791 (2005).

[18] Y. M. Cho, K. Kimm and J. H. Yoon, Mod. Phys. Lett. A 31, no. 09, 1650053 (2016); Phys. Lett. B 761, 203 (2016).

[19] J. Ellis, N. E. Mavromatos and T. You, Phys. Lett. B 756, 29 (2016); J. Ellis, V. Sanz and T. You, JHEP 1407, 036 (2014).

[20] M. Barriola and A. Vilenkin, Phys. Rev. Lett. 63, 341 (1989).

[21] A. S. Goldhaber, Phys. Rev. Lett. 63, 2158 (1989); S. H. Rhie and D. P. Bennett, Phys. Rev. Lett. 67, 1173 (1991); L. Perivolaropoulos, Nucl. Phys. B 375, 665 (1992); G. W. Gibbons, M. E. Ortiz, F. Ruiz Ruiz and T. M. Samols, Nucl. Phys. B 385, 127 (1992); M. Hindmarsh, Nucl. Phys. B 392, 461 (1993); G. Arreaga, I. Cho and J. Guven, Phys. Rev. D 62, 043520 (2000); A. Achucarro and J. Urrestilla, Phys. Rev. Lett. 85, 3091 (2000); R. Gregory and C. Santos, Class. Quant. Grav. 20, 21 (2003); S. B. Gudnason and J. Evslin, Phys. Rev. D 92, no. 4, 045044 (2015); A. Achucarro, B. Hartmann and J. Urrestilla, JHEP 0507, 006 (2005).

[22] A. K. Drukier and S. Nussinov, Phys. Rev. Lett. 49, 102 (1982).

[23] P. O. Mazur and J. Papavassiliou, Phys. Rev. D 44, 1317 (1991).

[24] N. E. Mavromatos and S. Sarkar, "Magnetic Monopoles from Global Monopoles in the presence of Kalb-Ramond Torsion," arXiv:1607.01315 [hep-th] (2016).

[25] Y. B. Zeldovich and M. Y. Khlopov, Phys. Lett. 79B, 239 (1978).

[26] C. T. Hill, Nucl. Phys. B 224, 469 (1983).

[27] V. K. Dubrovich, Grav. Cosmol. Suppl. 8N1, 122 (2002).

[28] A. Rajantie, Contemp. Phys. 53, 195 (2012).

[29] A. Rajantie, Phys. Today 69, no. 10, 40 (2016).

[30] L. N. Epele, H. Fanchiotti, C. A. Garcia Canal and V. Vento, Eur. Phys. J. C 56, 87 (2008); L. N. Epele, H. Fanchiotti, C. A. G. Canal and V. Vento, Eur. Phys. J. C 62, 587 (2009).

[31] L. N. Epele, H. Fanchiotti, C. A. G. Canal, V. A. Mitsou and V. Vento, Eur. Phys. J. Plus 127, 60 (2012).

[32] L. N. Epele, H. Fanchiotti, C. A. G. Canal, V. A. Mitsou and V. Vento, "Can the $750 \mathrm{GeV}$ enhancement be a signal of light magnetic monopoles?," arXiv:1607.05592 [hep-ph] (2016). 
[33] M. Kalliokoski, J. W. Lämsä, M. Mieskolainen and R. Orava, “Turning the LHC Ring into a New Physics Search Machine," arXiv:1604.05778 [hep-ex] (2016).

[34] B. Acharya et al. [MoEDAL Collaboration], JHEP 1608, 067 (2016).

[35] G. Aad et al. [ATLAS Collaboration], Phys. Rev. Lett. 109, 261803 (2012).

[36] G. Aad et al. [ATLAS Collaboration], Phys. Rev. D 93, 052009 (2016).

[37] B. Acharya et al. [MoEDAL Collaboration], Phys. Rev. Lett. 118, 061801 (2017).

[38] L. Patrizii and M. Spurio, Ann. Rev. Nucl. Part. Sci. 65, 279 (2015).

[39] H. Goldberg, Phys. Rev. Lett. 50, 1419 (1983) Erratum: [Phys. Rev. Lett. 103, 099905 (2009)]; J. R. Ellis, J. S. Hagelin, D. V. Nanopoulos, K. A. Olive and M. Srednicki, Nucl. Phys. B 238, 453 (1984).

[40] V. A. Mitsou, PoS PLANCK 2015, 085 (2015).

[41] J. R. Ellis, K. A. Olive, Y. Santoso and V. C. Spanos, Phys. Lett. B 565, 176 (2003).

[42] O. Buchmueller et al., Eur. Phys. J. C 72, 2243 (2012); Eur. Phys. J. C 74, 2922 (2014).

[43] T. Jittoh, J. Sato, T. Shimomura and M. Yamanaka, Phys. Rev. D 73, 055009 (2006) Erratum: [Phys. Rev. D 87, 019901 (2013)].

[44] S. Kaneko, J. Sato, T. Shimomura, O. Vives and M. Yamanaka, Phys. Rev. D 78, 116013 (2008) Erratum: [Phys. Rev. D 87, 039904 (2013)].

[45] J. R. Ellis, K. A. Olive and Y. Santoso, JHEP 0810, 005 (2008).

[46] J. L. Diaz-Cruz, J. R. Ellis, K. A. Olive and Y. Santoso, JHEP 0705, 003 (2007).

[47] K. Hamaguchi, M. M. Nojiri and A. de Roeck, JHEP 0703, 046 (2007).

[48] J. L. Feng, S. Iwamoto, Y. Shadmi and S. Tarem, JHEP 1512, 166 (2015).

[49] N. Arkani-Hamed and S. Dimopoulos, JHEP 0506, 073 (2005); G. F. Giudice and A. Romanino, Nucl. Phys. B 699, 65 (2004) Erratum: [Nucl. Phys. B 706, 487 (2005)].

[50] J. C. Pati and A. Salam, Phys. Rev. D 10, 275 (1974) Erratum: [Phys. Rev. D 11, 703 (1975)].

[51] R. N. Mohapatra and J. C. Pati, Phys. Rev. D 11, 566 (1975).

[52] G. Senjanovic and R. N. Mohapatra, Phys. Rev. D 12, 1502 (1975); R. N. Mohapatra and G. Senjanovic, Phys. Rev. D 23, 165 (1981).

[53] C. S. Aulakh, A. Melfo and G. Senjanovic, Phys. Rev. D 57, 4174 (1998); Z. Chacko and R. N. Mohapatra, Phys. Rev. D 58, 015003 (1998); C. S. Aulakh, K. Benakli and G. Senjanovic, Phys. Rev. Lett. 79, 2188 (1997).

[54] C. W. Chiang, T. Nomura and K. Tsumura, Phys. Rev. D 85, 095023 (2012).

[55] N. Arkani-Hamed, S. Dimopoulos and G. R. Dvali, Phys. Lett. B 429, 263 (1998); Phys. Rev. D 59, 086004 (1999); I. Antoniadis, N. Arkani-Hamed, S. Dimopoulos and G. R. Dvali, Phys. Lett. B 436, 257 (1998).

[56] L. Randall and R. Sundrum, Phys. Rev. Lett. 83, 4690 (1999).

[57] I. Antoniadis, Phys. Lett. B 246, 377 (1990); I. Antoniadis and K. Benakli, Phys. Lett. B 326, 69 (1994); I. Antoniadis, K. Benakli and M. Quiros, Phys. Lett. B 331, 313 (1994). 
[58] T. Appelquist, H. C. Cheng and B. A. Dobrescu, Phys. Rev. D 64, 035002 (2001).

[59] S. B. Giddings and S. D. Thomas, Phys. Rev. D 65, 056010 (2002).

[60] W. Fischler, “A Model for high-energy scattering in quantum gravity,” arXiv:hep-th/9906038 (1999).

[61] P. C. Argyres, S. Dimopoulos and J. March-Russell, Phys. Lett. B 441, 96 (1998); S. Dimopoulos and G. L. Landsberg, Phys. Rev. Lett. 87, 161602 (2001); G. L. Alberghi, R. Casadio and A. Tronconi, J. Phys. G 34, 767 (2007); M. Cavaglia, R. Godang, L. Cremaldi and D. Summers, Comput. Phys. Commun. 177, 506 (2007); D. C. Dai, G. Starkman, D. Stojkovic, C. Issever, E. Rizvi and J. Tseng, Phys. Rev. D 77, 076007 (2008).

[62] R. Casadio and B. Harms, Int. J. Mod. Phys. A 17, 4635 (2002).

[63] M. Cavaglia, Int. J. Mod. Phys. A 18, 1843 (2003); P. Kanti, Int. J. Mod. Phys. A 19, 4899 (2004).

[64] C. M. Harris, P. Richardson and B. R. Webber, JHEP 0308, 033 (2003).

[65] B. Koch, M. Bleicher and H. Stoecker, J. Phys. G 34, S535 (2007).

[66] S. Hossenfelder, B. Koch and M. Bleicher, “Trapping black hole remnants," hep-ph/0507140 (2005).

[67] T. Sjostrand, S. Mrenna and P. Z. Skands, JHEP 0605, 026 (2006).

[68] J. R. Ellis, N. E. Mavromatos and D. V. Nanopoulos, Gen. Rel. Grav. 32, 943 (2000); J. R. Ellis, N. E. Mavromatos and D. V. Nanopoulos, Phys. Lett. B 665, 412 (2008); J. R. Ellis, N. E. Mavromatos and M. Westmuckett, Phys. Rev. D 70, 044036 (2004); ibid. 71, 106006 (2005).

[69] G. Shiu and L. T. Wang, Phys. Rev. D 69, 126007 (2004).

[70] See, e.g., E. Witten, “Comments on string theory," arXiv:hep-th/0212247 (2002), and references therein.

[71] N. E. Mavromatos, S. Sarkar and A. Vergou, Phys. Lett. B 696, 300 (2011).

[72] N. E. Mavromatos, V. A. Mitsou, S. Sarkar and A. Vergou, Eur. Phys. J. C 72, 1956 (2012).

[73] S. Kasuya and M. Kawasaki, Phys. Rev. Lett. 85, 2677 (2000); S. Kasuya, M. Kawasaki and T. T. Yanagida, PTEP 2015, no. 5, 053B02 (2015); S. Abel and A. Kehagias, JHEP 1511, 096 (2015). 